\title{
Topics on Solid Angles
}

\author{
L. C. M. Amaral \\ Dallas, TX, USA \\ Email: py11l@yahoo.com
}

How to cite this paper: Amaral, L.C.M. (2019) Topics on Solid Angles. Journal of Applied Mathematics and Physics, 7, 2423-2439.

https://doi.org/10.4236/jamp.2019.710164

Received: September 19, 2019

Accepted: October 20, 2019

Published: October 23, 2019

Copyright (c) 2019 by author(s) and Scientific Research Publishing Inc. This work is licensed under the Creative Commons Attribution International License (CC BY 4.0).

http://creativecommons.org/licenses/by/4.0/

\begin{abstract}
The purpose of the present work is to derive some solutions for several solid angle cases via a fundamental formula which gives the solid angle for an isosceles triangle. From this formula the solid angle of pyramids is derived but, unlike other presentations, it is shown in a format similar to that of the well-known cone case. Besides the regular polygon cases (straight pyramids), solid angles of some other plane closed curves are calculated. The fundamental formula also leads to some interesting properties showing the not simple behavior of solid angles with the observer point on the curve itself, as it depends on how the observer arrived there. The question of the equi- $\Omega$ surfaces is also discussed and calculated in simple cases.
\end{abstract}

\section{Keywords}

Solid Angles, Pyramid Solid Angles, Electric Field Flux, Equi- $\Omega$ Surfaces

\section{Introduction}

When trying to calculate the electric field flux through windows on solids, one comes across with solid angle problems, in special related to pyramids. The same problem occurs when one uses radiation detectors with some noncircular windows to take into account the detection efficiency [1] [2].

As any $N$ side inscribable polygon is composed by a set of $N$ isosceles triangles, the solid angle for any pyramid with that base is simply the sum of the $N$ solid angles of those triangles, with the observer on the vertex of the pyramid. So, a natural path is to determine the solid angle $\Omega$ of an isosceles triangle with the observer point $\mathrm{P}$ on a vertical from the triangle vertex at a height $z$. The point $\mathrm{P}$ ' is the normal projection of $\mathrm{P}$ on the triangle vertex, that is, the vertex itself. The importance of the point $\mathrm{P}^{\prime}$ is that all calculations can be done on it instead of $\mathrm{P}$ directly, the solid angle seen by $\mathrm{P}$ being computed after by taking into account its height through the value of $z$. 
Normally solid angle for closed curves is shown when the observer point is in any position of the space but not on the curve itself. In the present article, the development of a Fundamental Formula, as performed in Section 2, leads to the possibility of calculating the solid angle for the observer on the curve. This is indeed the principal aim of the present work, with many consequences being pointed out and studied.

One more importance of $\mathrm{P}^{\prime}$ is clear when the observer is in any point of the curve plane, the plane of the curve $\left(\mathrm{P}^{\prime}=\mathrm{P}\right)$, including on the curve itself, when certain interesting properties of $\Omega$ arise. In special, on the curve, $\Omega$ presents a not simple behavior, that is, unlike on all other positions of $\mathrm{P}$ in the space, $\Omega$ cannot be defined on the curve only by its position. It is necessary to specify the path of $\mathrm{P}$ through which $\mathrm{P}$ reaches the curve.

This not simple scalar behavior also occurs with the problem of the internal angle of a circle arc and one chord as shown in Figure 1. The internal angle is a function of the position of the observer vertex on the plane but the end points of the chord. On these points, the internal angle is also a function of the path the observer reached them, as there are infinite arcs that have the same chord. In Figure 1 we see two different arcs $\mathrm{C} 1$ and $\mathrm{C} 2$ and the directions, the observer point arrived to the end $\mathrm{B}$ of the chord $\mathrm{AB}$ (that are the internal angles themselves). This shows that, on one end of the segment, the internal angle is not only a function only of the position, but also a function of how it arrives on that end.

\section{The Fundamental Formula}

The solid angle through it an observer on point $\mathrm{P}$ sees an isosceles triangle is given by the fundamental formula, Equation (1) below. This was derived by simple double integration. Many consequences of the fundamental formula and several applications of it are shown along with this paper.

Refer to Figure 2. Let $\mathrm{BC}=L$ be the base of the isosceles triangle P'BC and with a distance $z$ from the plane $\mathrm{X}-\mathrm{Y}$. The point $\mathrm{D}$ is then on that plane. To calculate the solid angle $\Omega$ the origin sees the triangle, we have:

$$
\mathrm{d} \Omega=\left(\hat{\mathrm{z}} \cdot \hat{\mathrm{r}} / r^{2}\right) \mathrm{d} S
$$

with $\hat{\mathrm{z}}$ the unitary vector normal to $\mathrm{d} S$ and $\hat{\mathrm{r}}$ the unitary vector in the direction of $r$. The position of a point on the line $\mathrm{P}^{\prime}-\mathrm{C}$ is given by $y=x \cdot \operatorname{tg}(\varphi / 2)$ and on the line P'-B by $y=-x \cdot \operatorname{tg}(\varphi / 2)$. The limits of $x$ are 0 and $p$, or $L /[2 \tan (\varphi / 2)]$.

The solid angle is given by:

$$
\Omega=z \cdot \int_{0}^{L /[2 \cdot \operatorname{tg}(\varphi / 2)]} \mathrm{d} x \cdot \int_{-x \cdot \operatorname{tg}(\varphi / 2)}^{x \cdot \operatorname{tg}(\varphi / 2)} \mathrm{d} y / r^{3}
$$

The result of this integral, replacing $L$ by $2 \cdot z \cdot \sin (\varphi / 2) \tan \theta$ is:

$$
\Omega=\varphi-2 \arctan [\cos \theta \tan (\varphi / 2)]
$$

Equation (1) is the Fundamental Formula and shows the solid angle the observer in $\mathrm{P}$ sees the isosceles triangle $\mathrm{P}$ ' $\mathrm{BC}$. 


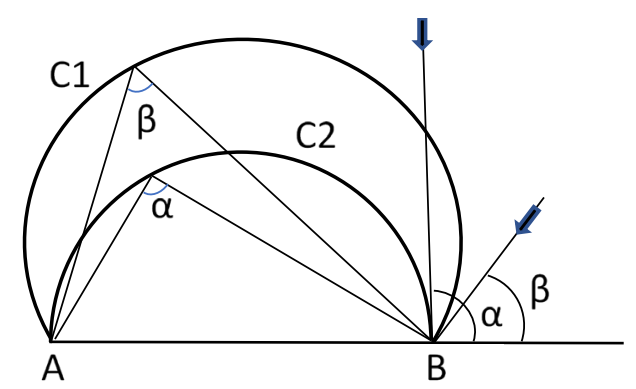

Figure 1. Internal angle problem.

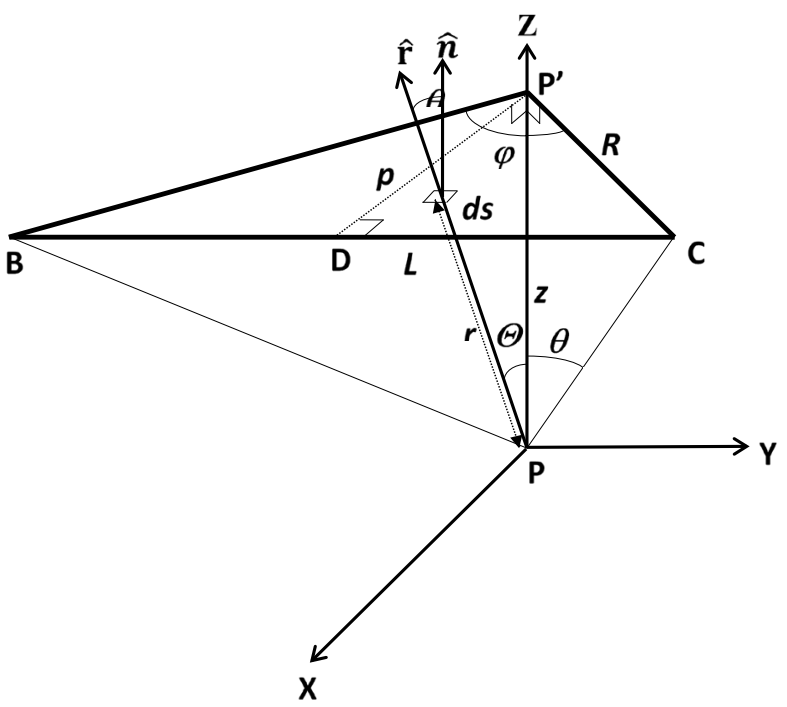

Figure 2. Derivation of the Fundamental Formula.

\section{The Observer in the Plane of the Triangle}

When the observer $\mathrm{P}$ is in the plane of the isosceles triangle, that is, $\mathrm{P}$ coinciding with $\mathrm{P}$ ', the solid angle is obviously $2 \pi$ with $\mathrm{P}$ within the triangle and 0 with the $\mathrm{P}$ is outside it. If $\mathrm{P}$ is exactly on the vertex, the solid angle depends on how $\mathrm{P}$ reaches the vertex. If $\mathrm{P}$ comes from outside the triangle the solid angle is 0 and from inside, the solid angle is $2 \pi$. If $\mathrm{P}$ comes from the space not on the triangle plane, the solid angle depends on the angle of arrival to the vertex. If $\mathrm{P}$ comes to P' through the z-axis (see Figure 2), the solid angle is got by making $z$ equal to 0 in Equation (1). The result is:

$$
\Omega=\varphi
$$

Equation (2) has some consequences. Suppose we have a general plane closed curve as that of Figure 3 with $\mathrm{P}$ on the vertex of an angular region of the curve. We divide the curve into two parts such that we get an isosceles triangle ' $a$ ' with ' $b$ ' being the rest of the figure. The solid angle seen by $\mathrm{P}$ is the sum of the solid angle of ' $a$ ' with that of ' $b$ '. As $P$ is on the plane of the curve and outside ' $b$ ', the ' $b$ ' corresponding solid angle is 0 . For 'a' the solid angle is $\varphi$ and so the total solid angle. Then the total solid angle is independent of the general shape of the curve but only dependent of the angle $\varphi$. 


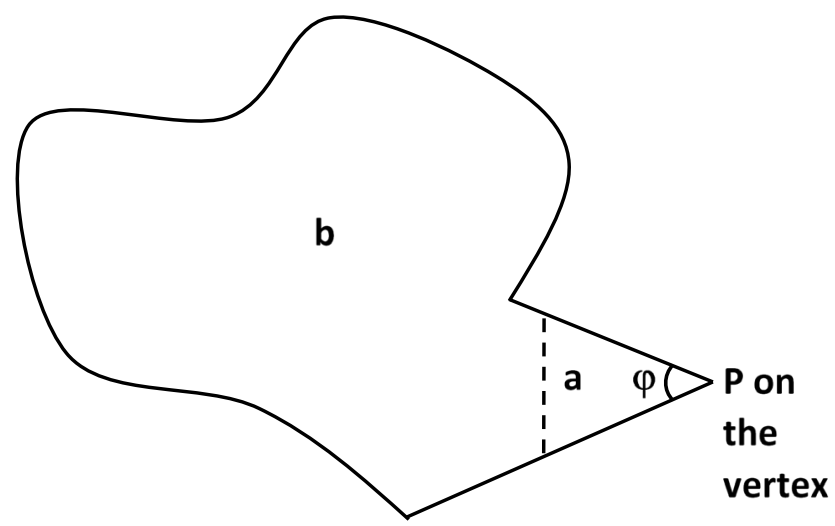

Figure 3. The observer on the vertex of an angular part of a curve.

When $\mathrm{P}$ is on a straight or smooth (not angular) part of a curve, $\varphi=\pi$ and, so, the solid angle is also $\pi$, independently of the rest of the curve, as shown in Figure 4. This is the case of the circle which that is useful in the study of the solid angle of cones.

We must remember that all these cases have the observer $\mathrm{P}$ on the plane of the curve $(z=0)$. The following analysis confirms the validity of Equation (2).

As in Figure 5, let's have a closed curve of arbitrary shape and with $\mathrm{P}$ in any position of the plane of the curve and within it. If we divide the figure into $N$ arbitrary angles $\alpha_{i}$, the total solid angle $\Omega$ will be the sum $\sum_{i}$ of the partial solid angles $\Omega_{i}$. As we saw before with Equation (2), $\Omega_{i}=\alpha_{i}$, so, as we have $\sum_{i} \alpha_{i}=2 \pi$, we get $\Omega=2 \pi$ as expected.

\section{Application of the Fundamental Formula}

\subsection{The Solid Angle of Right Pyramids with P' at Its Center}

If the closed plane curve is an inscribable $N$ side polygon, it can be divided into $N$ isosceles triangles, the solid angle of the straight pyramid having this polygon as its base is a sum of the $N$ results of Equation (1), one for each triangle. The particular case of this situation is the regular $N$ side polygon, creating a regular pyramid. As $\varphi$ is $2 \pi$ divided by $N$, we get the pyramid solid angle by multiplying Equation (1) by $N$. The resulting formula, Equation (3), is conveniently written with a similar shape of the well-known cone case, unlike other publications [3].

$$
\Omega=2 \pi\{1-(N / \pi) \arctan [\cos \theta \tan (\pi / N)]\}
$$

Equation (3) is useful to solve many geometric problems, as we see in item 5.

Some of these problems are classical ones that can be solved in a much simpler and quicker manner using Equation (3) and some are newly proposed ones.

\subsection{The Solid Angle of a General Triangle with P' on Its Vertex}

Let's consider the case with the observer $\mathrm{P}$ on the vertical over the vertex of a general triangle as in Figure 6. Its vertices are located in points $\mathrm{P}$ ', $\mathrm{S}$ and $\mathrm{Q}$ and $\mathrm{P}$ 


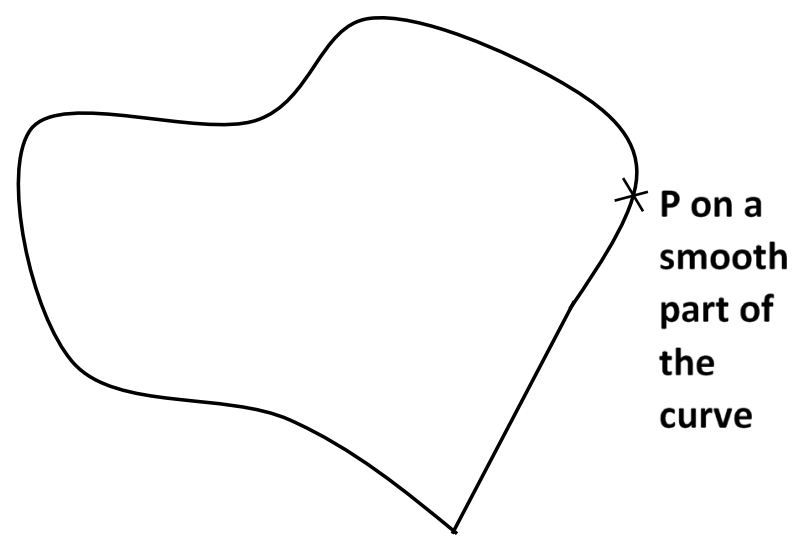

Figure 4. The observer on a smooth part of a curve.

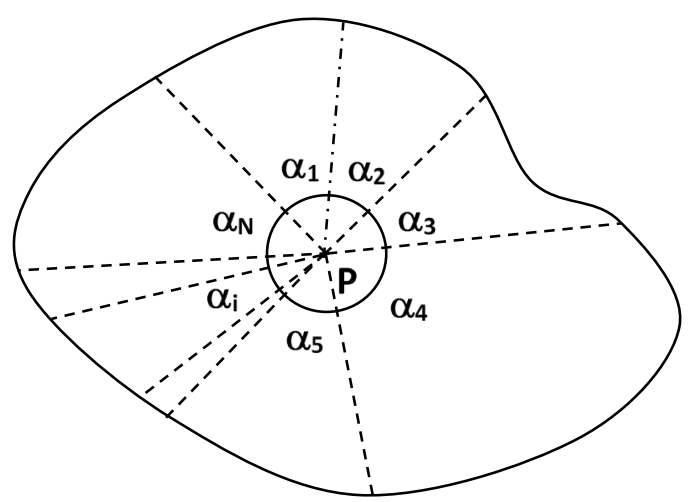

Figure 5. One of Equation (2) validity confirmation.

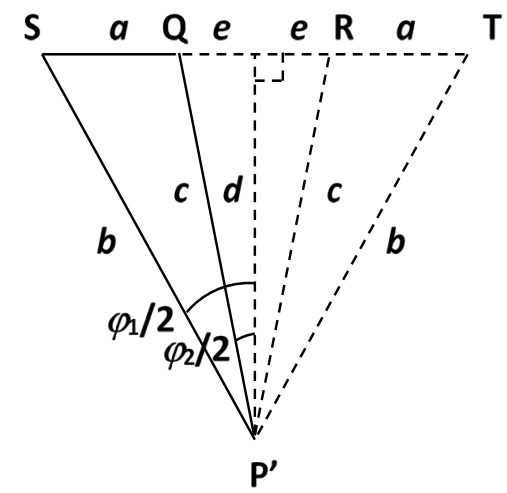

Figure 6. The case of $P^{\prime}$ on the vertex of a general triangle.

is at a height $\mathrm{z}$ from the triangle plane. The lengths $\mathrm{a}, \mathrm{b}, \mathrm{c}$ and $\mathrm{z}$ are given and $\mathrm{a}$ is the side not containing the point $\mathrm{P}^{\prime}$. With $\mathrm{b}$ being the biggest between the two other sides, we build two isosceles triangles P'QR and P'ST. The segment e completes both central right triangles. This processes for determining solid angles of geometric figures are not exclusive of triangles,

The solid angle relative to the original triangle P'SQ is obviously given by:

$$
\Omega=\left(\Omega_{1}-\Omega_{2}\right) / 2
$$


where $\Omega_{1}$ and $\Omega_{2}$ are the solid angles relative to the triangles P'ST and P'QR respectively.

With:

$$
K=4\left(a^{2} b^{2}+a^{2} c^{2}+b^{2} c^{2}\right)-\left(a^{2}+b^{2}+c^{2}\right)^{2}
$$

We have:

$$
\begin{aligned}
& \tan ^{2}\left(\varphi_{1} / 2\right)=\left(b^{2}-c^{2}+a^{2}\right)^{2} / K \\
& \tan ^{2}\left(\varphi_{2} / 2\right)=\left(b^{2}-c^{2}-a^{2}\right)^{2} / K
\end{aligned}
$$

With some manipulation and using Equation (1), we get for the solid angle:

$$
\begin{aligned}
& \Omega=\arctan \left\{\left(b^{2}-c^{2}+a^{2}\right) / \sqrt{\left[4\left(a^{2} b^{2}+a^{2} c^{2}+b^{2} c^{2}\right)-\left(a^{2}+b^{2}+c^{2}\right)^{2}\right]}\right\} \\
& -\arctan \left\{z\left(b^{2}-c^{2}+a^{2}\right) / \sqrt{\left\{\left[4\left(a^{2} b^{2}+a^{2} c^{2}+b^{2} c^{2}\right)-\left(a^{2}+b^{2}+c^{2}\right)^{2}\right]\left(b^{2}+z^{2}\right)\right\}}\right\} \\
& -\arctan \left\{\left(b^{2}-c^{2}-a^{2}\right) / \sqrt{\left[4\left(a^{2} b^{2}+a^{2} c^{2}+b^{2} c^{2}\right)-\left(a^{2}+b^{2}+c^{2}\right)^{2}\right]}\right\} \\
& +\arctan \left\{z\left(b^{2}-c^{2}-a^{2}\right) / \sqrt{\left\{\left[4\left(a^{2} b^{2}+a^{2} c^{2}+b^{2} c^{2}\right)-\left(a^{2}+b^{2}+c^{2}\right)^{2}\right]\left(c^{2}+z^{2}\right)\right\}}\right\}
\end{aligned}
$$

The expression above is rather complicated but is a real and exact solution of the proposed problem.

As a test of Equation (4), we make $c=b$, that means, the triangle is isosceles. We get $\Omega=\varphi-2 \arctan [\cos \theta \tan (\varphi / 2)]$, that is the Equation (1), as expected.

If $\mathrm{P}^{\prime}$ is not on the vertex of the triangle, we can create three new triangles $\alpha \beta \mathrm{P}^{\prime}$, $\beta \chi \mathrm{P}^{\prime}$ and $\alpha \chi \mathrm{P}^{\prime}$ as in Figure 7 and solve the problem for these three general triangles as shown before in the present section. The sought solid angle $\Omega(\alpha \beta \chi)$ is given by:

$$
\Omega(\alpha \beta \chi)=\Omega\left(\alpha \beta \mathrm{P}^{\prime}\right)+\Omega\left(\beta \chi \mathrm{P}^{\prime}\right)-\Omega\left(\alpha \chi \mathrm{P}^{\prime}\right)
$$

This solution, although shown for the triangle case, it is perfectly valid for any polygon, as one can create a number of suitable triangles to calculate the solid angle.

Normally it is possible to create $N$ triangles for the case of a general polygon with $N$ sides. This leads to the solid angle for a general pyramid with a general polygonal base [4]. The process may be laborious due the number of involved triangles but leads to precise results. The next section just deals with the special case of rectangles, that are simpler to calculate as it involves only isosceles triangles that are well treated in Section 2.

Reference [5] shows another approach to the solid angle for a general triangle.

\subsection{The Solid Angle of a General Rectangle}

It is possible to solve this problem using Section 4.2 choosing the rectangle as the general polygon, but the following solution is simpler and immediate. 


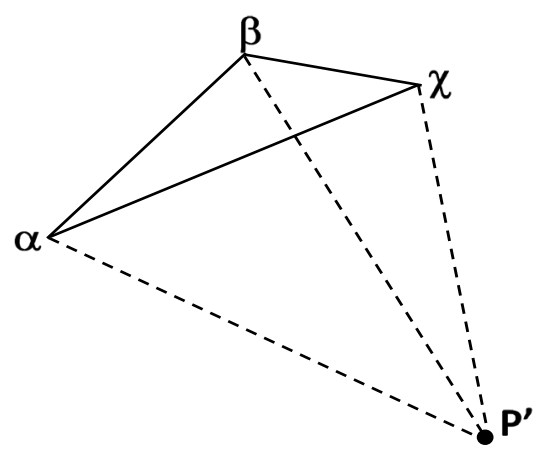

Figure 7. The case of a general triangle.

A rectangle is dividable into 4 isosceles triangles equal two by two with sides $2 \mathrm{~A}$ and $2 \mathrm{~B}$. Then, if $\mathrm{P}^{\prime}$ is on the center of a rectangle, the problem of the solid angle for such a pyramid is solved by using Equation (1) two times, one for each type of triangle, summing their results and multiple by two. The result is shown by Equation (5) below:

$$
\begin{aligned}
\Omega= & 2 \pi-4 \arctan \left\{z A /\left[B\left(A^{2}+B^{2}+z^{2}\right)^{1 / 2}\right]\right\} \\
& -4 \arctan \left\{z B /\left[A\left(A^{2}+B^{2}+z^{2}\right)^{1 / 2}\right]\right\}
\end{aligned}
$$

When $\mathrm{P}^{\prime}$ is not on the center, for example, the situation is that of Figure 8, where the rectangle is $\alpha \beta \chi \varepsilon$ of sides 2.a and 2.b. To solve this case, we trace two central lines containing P' and parallel to the rectangle sides and create three more rectangles, $\gamma \delta \lambda \eta, \rho \sigma \zeta \psi$ and $\mu \nu \xi \kappa$, the central lines three mirror images of the first one. As we see in Figure 8, the problem now is reduced to calculate the solid angles of four rectangle with P' common to all. These rectangles are: $\alpha \delta \zeta \kappa, \beta \gamma \psi \xi$, $\mu \varepsilon \eta \sigma$ and $\chi \lambda \rho \nu$, with the respective solid angles $\Omega(\alpha \delta \zeta \kappa), \Omega(\beta \gamma \psi \xi)$, $\Omega(\mu \varepsilon \eta \sigma)$ and $\Omega(\chi \lambda \rho v)$. The solid angle $\Omega$ of the original rectangle is given by $\Omega=[\Omega(\alpha \delta \zeta \kappa)-\Omega(\beta \gamma \psi \xi)-\Omega(\mu \varepsilon \eta \sigma)+\Omega(\chi \lambda \rho v)] / 4 \quad$ (The last term is the compensation for the central rectangle $\chi \lambda \rho v$ that was accounted two times with the rectangles $\beta \gamma \psi \xi$ and $\mu \varepsilon \eta \sigma)$.

Using Equation (2) and Equation (4), after some manipulation we get for the solid angle of the original rectangle:

$$
\begin{aligned}
\Omega= & \arctan \left\{\left[(x+a)^{2}+(y-b)^{2}+z^{2}\right]^{1 / 2} z /[(x+a)(y-b)]\right\} \\
& +\arctan \left\{\left[(x-a)^{2}+(y+b)^{2}+z^{2}\right]^{1 / 2} z /[(x-a)(y+b)]\right\} \\
& -\arctan \left\{\left[(x+a)^{2}+(y+b)^{2}+z^{2}\right]^{1 / 2} z /[(x+a)(y+b)]\right\} \\
& -\arctan \left\{\left[(x-a)^{2}+(y-b)^{2}+z^{2}\right]^{1 / 2} z /[(x-a)(y-b)]\right\}
\end{aligned}
$$

As before, the expression is complicated but exact. It is possible to condensate yet more Equation (6), but the result does not seem to be more convenient to use. 


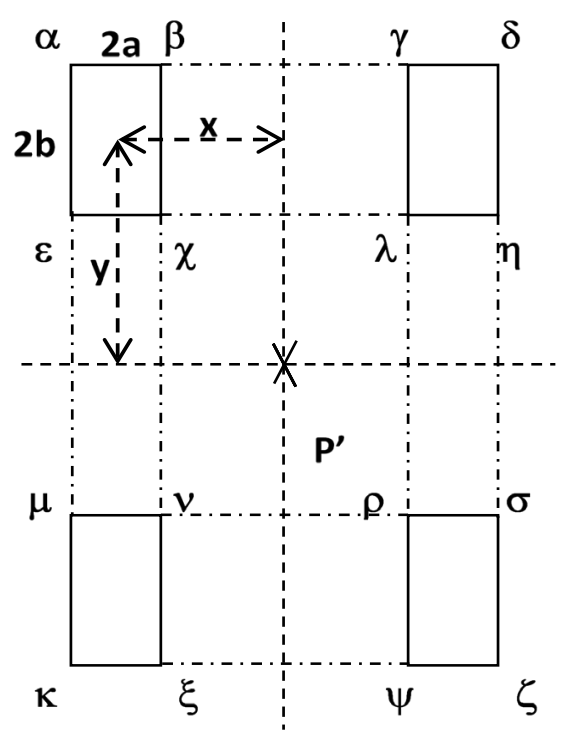

Figure 8. The case of a general rectangle.

One test may be performed on Equation (6) by making $x=a, y=b$, that is, putting $\mathrm{P}$ ' on the vertex $\chi$. Choosing $a=b$ and $z=2 a$, the point $\mathrm{P}$ will be seeing one quarter of the face of a cube and situated at its center, that is, $\Omega=\pi / 6$. Multiplying this by four, we get $\Omega=2 \pi / 3$, the solid angle of the face of a cube (as multiplying this by six we get $4 \pi$ ).

\subsection{The Solid Angle of an Infinite Long Band}

Suppose we have an infinite long band with width $p$ as in Figure 9(a). To get the solid angle the point $\mathrm{P}$ sees the infinite band, we draw an isosceles triangle as in Figure 9(b), keeping the distance $p$ and getting the limit when $L$ tends to infinity. Using Equation (1) and getting the limit for $L$, we have:

$$
\Omega=\pi-2 \arctan (z / p)
$$

Making $Z=0$ in Equation (5), we get $\Omega=\pi$, in agreement with Equation (2), as expected.

If $\mathrm{P}$ is on the middle of a band of width $p$, we simply double the result of Equation (7), replacing $p$ by $p / 2$ :

$$
\Omega=2 \pi-4 \operatorname{tg}^{-1}(2 z / p)
$$

So, using Figure 10 and the Equation (7), we can obtain the solid angle for any point $\mathrm{P}$ outside an infinite band with width $p$. Now, we have two bands, one with width $y-p / 2$ and another with width $y+p / 2$. The subtraction of the solid angles $\Omega_{1}$ and $\Omega_{2}$ given by Equation (7) corresponding to both bands gives us the solid angle that $\mathrm{P}$ sees the original band with width $p$ :

$$
\Omega_{1}=\pi-2 \arctan [z /(y+p / 2)] \text { and } \Omega_{2}=\pi-2 \arctan [z /(y-p / 2)]
$$

$\Omega=\Omega_{1}-\Omega_{2}$, therefore: 


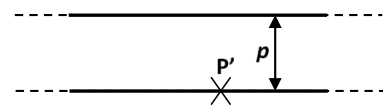

(a)

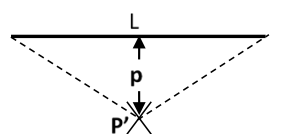

(b)

Figure 9. (a): The infinite band with the observer on its edge; (b): The solution of this case.

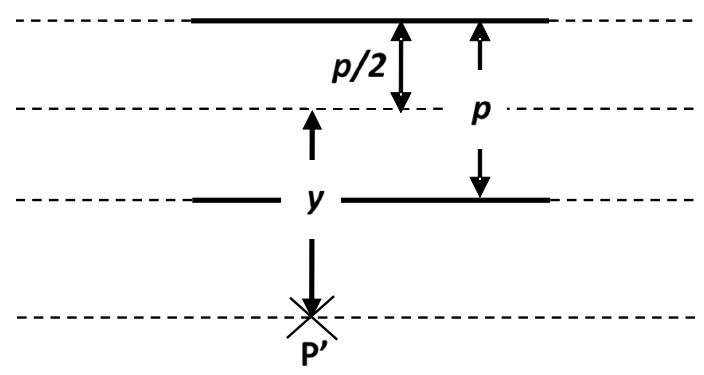

Figure 10. $\mathrm{P}^{\prime}$ outside an infinite band.

$$
\Omega=2\{\arctan [z /(y-p / 2)]-\arctan [z /(y+p / 2)]\}
$$

Equation (9) shows the solid angles seen by $\mathrm{P}$ in any point of the space but those on the band plane, where it is valid only for points with $\mathrm{P}^{\prime}$ is outside the band. If we make $z=0$ on Equation (9), we get $\Omega=0$.

The problem on the band plane is that $\Omega$ is discontinuous on it. To by-pass this problem, we use Figure 11 where we have two bands with width $p / 2-y$ and $p / 2+y$. The solid angle $\Omega$ seen by $\mathrm{P}$ with $\mathrm{P}^{\prime}$ inside the band is the sum of the solid angle corresponding to the two bands. Using Equation (7) we get:

$$
\Omega_{1}=\pi-2 \arctan [z /(p / 2+y)] \text { and } \Omega_{2}=\pi-2 \arctan [z /(p / 2-y)]
$$

$\Omega=\Omega_{1}+\Omega_{2}$, therefore:

$$
\Omega=2 \pi-2\{\arctan [z /(y-p / 2)]+\arctan [z /(y+p / 2)]\}
$$

Equation (10) is valid for all points in the space but those on the band plane, where it is valid only for points with $\mathrm{P}^{\prime}$ is inside the band. If we make $z=0$ on Equation (10) we get $\Omega=2 \pi$.

The solid angle seen by $\mathrm{P}$ when it is on the edge of the band depends on how $\mathrm{P}$ approaches the edge. Refer to Figure 12. For the general case, we consider $\mathrm{P}$ reaches the edge along the dotted line, that means, with constant $\varphi$. From the figure we may write $y-p / 2=x, y+p / 2=x+p, z / x=\tan \varphi$ and we can rewrite Equation (9) as:

$$
\Omega=2 \varphi-2 \arctan [x \tan \varphi /(p+x)]
$$

To obtain the solid angle $\Omega_{\varphi}$ with P reaching the edge under an angle $\varphi$, we calculate the limit of the Equation (11) when $\mathrm{x}$ tends to zero (keeping constant $\varphi)$ :

$$
\Omega_{\varphi}=2 \varphi
$$




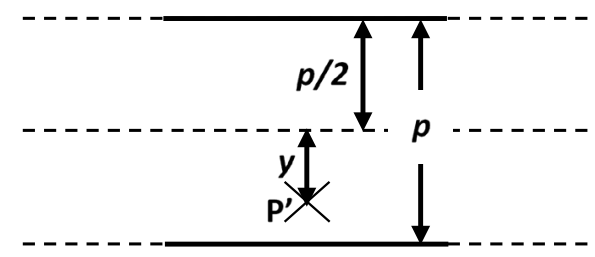

Figure 11. P' on the infinite band.

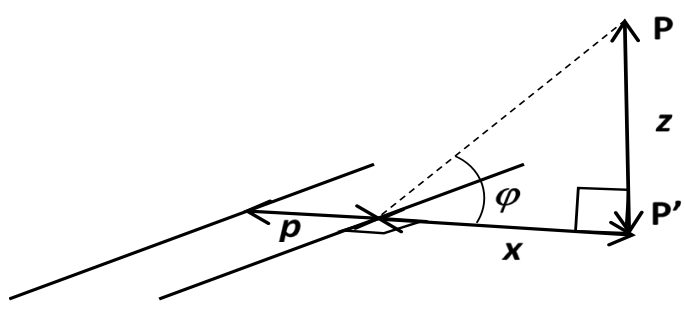

Figure 12. $\mathrm{P}$ approaching the edge with an angle $\varphi$.

This is independent of $p$ (for $p \neq 0$; if $p=0, \Omega=0$ ). So, suppose $\mathrm{P}$ goes to the edge of the band. When $\varphi=0$, that is, $\mathrm{P}^{\prime}$ originally off the band, then $\Omega_{\varphi}=0$, as expected; if $\varphi=\pi$ and $y<p$, that is, P' originally within the band, $\Omega_{\varphi}=2 \pi$, also as expected; if $\varphi=\pi / 2$, that is, P' originally on the edge of the band, $\Omega_{\varphi}=\pi$, as expected for any smooth curve case. This dependence of $\Omega_{\varphi}$ on the arriving angle of $\mathrm{P}$, in the case of $\mathrm{P}$ ' on the edge, is a very common fact to all curve shapes to be observed by $\mathrm{P}$. This shows the not simple behavior of $\Omega_{\varphi}$ for this case, where the position of $\mathrm{P}$ is not enough to determine the solid angle (we need another parameter: the arriving angle).

Note that, in Figure 12, the movement of $\mathrm{P}$ to reach the edge is on the plane perpendicular to the band. This is not a lack of generality because the component of the movement parallel to the band does not alter the solid angle as the band length is infinite. We have indeed a cylindrical symmetry.

\subsection{Surface Equi- $\Omega$ of an Infinite Band}

An Equi- $\Omega$ surface is one formed by observation points where the solid angle is the same. Those surfaces are got by fixing the value of $\Omega\left(\Omega=\Omega_{0}\right)$. Normally it is hard to determine for the most of the curves because of mathematical difficulties that arise during the calculus.

One of the simple ones is that related to the infinite band case discussed in Section 4.4. We get the surface for this case making $\Omega=\Omega_{0}$ in Equation (9) and writing an expression relating the variables $z$ and $y$. Equation (9) may be written as:

$$
z^{2}+y^{2}-p z / \tan \left(\Omega_{0} / 2\right)-p^{2} / 4=0
$$

Equation (13) represents an arc of a circle with coordinates of the center $y_{0}=0, \quad z_{0}=p /\left[2 \tan \left(\Omega_{0} / 2\right)\right]$ and radius $R=p\left[1+\cot \left(\Omega_{0} / 2\right)\right]^{1 / 2} / 2$. The overall curve, composed by those arcs of circle, is symmetrical regarding the 
plane of the band center straight line as in Figure 13. The curve is, indeed, the cross section of an infinite cylinder.

Let us choose two examples of such curves. The first one is the case when the solid angle $\Omega_{0}=\pi$ and $\tan \left(\Omega_{0} / 2\right) \rightarrow \infty$. Equation (13) is written as:

$$
z^{2}+y^{2}-p^{2} / 4=0
$$

The circle has $y_{0}=z_{0}=0$ and radius $R=p / 2$, as in Figure 14. The diameter line is the cross section of the band with width $p$. The band is normal to the paper. Note that the angle the center sees the band edges is just the solid angle value $\Omega_{0}$. This is because the circle is the geometric locus of half of $\Omega_{0}$ or $\varphi$. The angle to the right of the figure is $\varphi$ of Equation (12). It is the angle through which $\mathrm{P}$ arrives at the edge of the band, that is, the angle of the tangent of the curve at the edge of the band. This is confirmed by the derivative $d z / d y$ of the Equation (14) when $z=0$ :

$$
\mathrm{d} z / \mathrm{d} y=\tan (\varphi)=y / z
$$

When $z \rightarrow 0, \tan (\varphi) \rightarrow \infty$ or $\varphi=\pi / 2$.

The second example is when the solid angle is $\Omega_{0}=\pi / 2$ and $\tan \left(\Omega_{0} / 2\right)=1$. Equation (13) is now:

$$
z^{2}+y^{2}-p z-p^{2} / 4=0
$$

The first circle has its center at $y_{0}=0, z_{0}=p / 2$ and $R=p / \sqrt{2}$. The overall curve is composed by two symmetrical arcs of circle as shown in Figure 15. The equi- $\Omega$ surface then is an infinite cylinder with the cross section of that figure. Note that the center angle, as in the first example, has the value $\Omega_{0}$. The circle is the geometric locus of half of $\Omega_{0}$ or $\varphi$. As the first case, the angle to the right of the figure is $\varphi$ of Equation (12). It is the angle through which the point $P$ arrives on the edge of the band, that is, the angle of the tangent of the curve at the edge. This can be confirmed by the derivative $\mathrm{d} z / \mathrm{d} y$ of the Equation (15) at the edge or when $z=0$ and $y=p / 2$. This leads to $\varphi=\pi / 4$.

For the case where $\Omega_{0}=0$, the locus of $\mathrm{P}$ is the band plane with P' outside it and up to the of infinity, as shown in Figure 16.

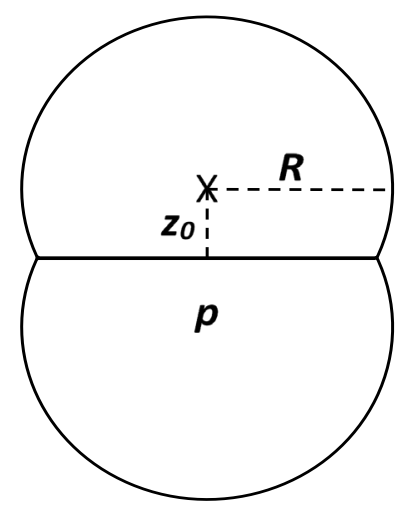

Figure 13. The Equi- $\Omega$ Surface for the infinite band case. 


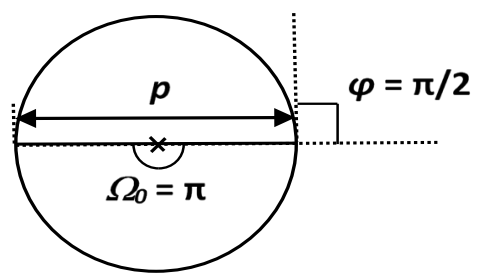

Figure 14. The infinite band case for $\varphi=\pi / 2$.

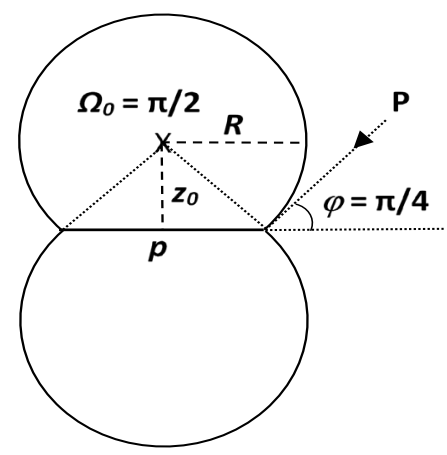

Figure 15. Cross section of equi- $\Omega$ surface of infinite band case for $\varphi=\pi / 4$.

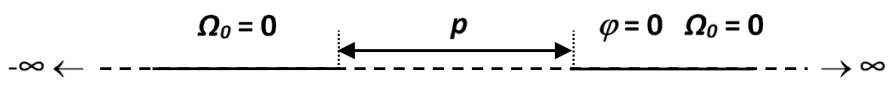

Figure 16. Cross section of the equi- $\Omega$ surface for the infinite band case with $\varphi=0$.

For the case where $\Omega_{0}=2 \pi$, the locus of $\mathbf{P}$ is the band plane for $\mathbf{P}^{\prime}$ inside it as shown in Figure 17.

\subsection{Observer on the Vertex of an Isosceles Triangle Arriving from any Direction}

We want to get the solid angle relative to the triangle $A B C$ of Figure 18 when $\mathrm{P}$ is on the point $\mathrm{C}$ arriving to it through the direction of the arrow at right part of that figure.

From the triangle ACP', at left part of Figure 18. We have:

$$
b^{2}=a^{2}+c^{2}+2 a c \cos (\varphi / 2)
$$

The solid angle $\Omega_{\gamma}$ for the triangle ABC is the difference between the solid angles for the isosceles triangle $\mathrm{ABP}$ ' and for the two identical triangles $\mathrm{ACP}$ ' and BCP':

$$
\Omega_{\gamma}=\Omega_{\mathrm{ABP}^{\prime}}-2 \Omega_{\mathrm{ACP}^{\prime}}
$$

$\Omega_{\mathrm{ABP}}$ is given by Equation (1) with $\varphi$ replaced by $\beta$ and, as $\gamma$ is constant during the movement of P, $z$ is replaced by $c \tan \gamma$ and we make $c$ tending to zero. $\Omega_{\mathrm{ACP}}$ is given by Equation (4) where $b^{2}$ is replaced by its value of Equation (16). The result is: 


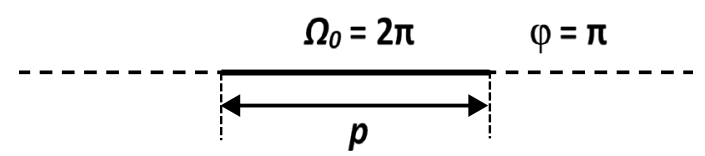

Figure 17. Cross section of the equi- $\Omega$ surface for the infinite band case with $\varphi=\pi$.

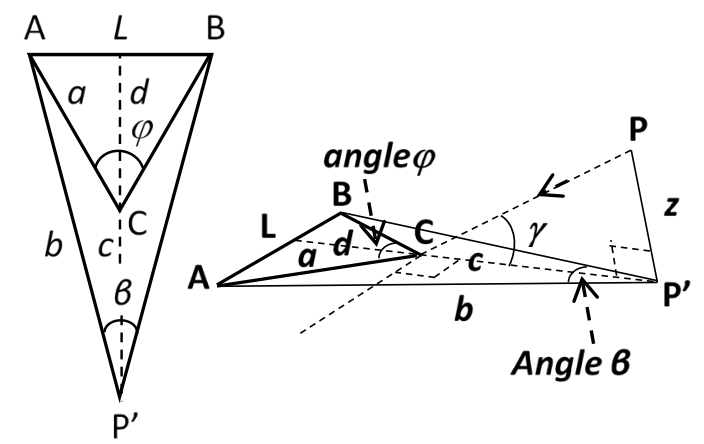

Figure 18. Solid Angle for an Isosceles Triangle with the Observer $\mathrm{P}$ at any position arriving through the arrow.

$$
\begin{aligned}
& \Omega_{\mathrm{ACP}^{\prime}}=\arctan \{[a+c \cos (\varphi / 2)] /[c \sin (\varphi / 2)]\} \\
& -\arctan \{[a+c \cos (\varphi / 2)] \tan \gamma\} /\left\{\sin (\varphi / 2)\left[a^{2}+2 a c \cos (\varphi / 2)+c^{2}(1+\tan \gamma)\right]^{1 / 2}\right\} \\
& -\arctan [\cot (\varphi / 2)] \\
& +\arctan \left\{c \cot (\varphi / 2) \tan \gamma /\left[a^{2}+2 a c \cos (\varphi / 2)+c^{2}(1+\tan \gamma)\right]^{1 / 2}\right\}
\end{aligned}
$$

The observer $\mathrm{P}$ approaches the point $\mathrm{C}$ if we get the limit of Equation (18) when $c$ tends to zero with the angle $\gamma$ kept constant. If $\Omega_{\gamma}$ is the resulting solid angle, we get:

$$
\Omega_{\gamma}=2 \arctan [\tan \gamma / \sin (\varphi / 2)]
$$

We can perform some confirmations of the above results. Suppose a smooth curve, that means, $\varphi=\pi$. For $\gamma=0$, using Equation (19), we get $\Omega_{0}=0$, as expected, because $\mathrm{P}$ initially is on the triangle plane, outside the triangle, and comes to the vertex. For $\gamma=\pi / 2$, we get $\Omega_{\pi / 2}=\varphi$, matching the result of Equation (2). For $\gamma=\pi$, we get $\Omega_{\pi}=2 \pi$, as expected, because $\mathrm{P}$ is on the triangle plane, within the triangle, and comes to the vertex. In Equation (19), if we put $\varphi=\pi \quad$ (the case of smooth curves on $\left.\mathrm{P}^{\prime}\right)$, we get:

$$
\Omega_{\gamma}=2 \gamma
$$

Equation (20) shows that, for any smooth curve. the solid angle for the curve seen by a point $\mathrm{P}$ on the curve and arriving at it through a constant angle $\gamma$, is just $2 \gamma$. This confirms the result of Equation (12), for the infinite band case. This result is also valid for the common cone case, as we see next. 


\section{Notes on Equi- $\Omega$ Surface for the Simple Cone Case}

The surface equi- $\Omega$ for the simple cone case has clearly rotational symmetry but it is not a spherical surface. A circumference of radius $\mathrm{R}$ is the base of a cone with height $h$ as Figure 19(a) shows. The surface is vertically flattened, with the vertical radius $R_{V}$ smaller than the horizontal one $R_{H}$ (it is not a sphere) and it is symmetrical related to the circumference plane. The observer $\mathrm{P}$ is reaching the point A of the base circumference through a constant angle $\gamma$. As a circumference is a smooth curve, the solid angle $\Omega$ seen by $\mathrm{P}$ when it arrives point A is $2 \gamma$, according Equation (21). If the surface is the equi- $\Omega$ one, the solid angle of the cone with angle $\theta$ must be also $\Omega$. So, we must have:

$$
2 \pi(1-\cos \theta)=2 \gamma
$$

or

$$
\cos \theta=1-\gamma / \pi
$$

If we suppose that the equi- $\Omega$ surface is spherical, we have the situation of Figure 19(b). By Equation (21), we must have $\Omega=\pi$, but, by the solid angle formula for the cone,

$\Omega=2 \pi[1-\cos (\pi / 4)]$ or $\Omega=\pi(1-\sqrt{2})$, two different values showing that the surface cannot be a sphere. For $\Omega=\pi, \theta$ must be $\pi / 3$ and not $\pi / 4$, showing the flatness of the surface. The equi- $\Omega$ surface equation with a general position of the observer $\mathrm{P}$ for the case of a circular base, that is, a general cone (not necessarily right), leads to a differential equation $\mathrm{d} \Omega=(\mathrm{d} \Omega / \mathrm{d} \varphi) \mathrm{d} \varphi$ with the function $\mathrm{d} \Omega / \mathrm{d} \varphi$ to much complex to be integrated. Numerical integrations are possible, but they do not lead to general formulas to solve the problem.

\section{Some Applications of Solid Angles of Right Regular Pyramids}

\subsection{Flux of Electric Field of a Point Charge through Faces of Polyhedrons}

Figure 20 shows a non-regular polyhedron with 12 faces of two types, four squares and eight equilateral triangles. As we have two types of faces, there are two different defined solid angles seen by the center, $\Omega_{s}$ and $\Omega_{t}$ (the indexes $s$ and $t$ stand for square and triangle respectively). The total flux $\Phi$ of a point charge $Q$ at the center of the polyhedron is:

$$
\Phi=\Omega Q /\left(4 \pi \varepsilon_{0}\right)
$$

where $\Omega$ is the solid angle by which the charge sees the face. With little geometric observation, the square base pyramid with the vertex at the solid center has $\cos \theta=1 / \sqrt{2}$. So, by using Equation (3), the solid angle $\Omega_{s}$ for a square face is given by:

$$
\Omega_{s}=2 \pi\{1-(4 / \pi) \arctan [(1 / \sqrt{2}) \tan (\pi / 4)]\}=1.3593
$$

As there are six square faces and eight triangular ones, the solid angle $\Omega_{t}$ for one triangular face is given by: 


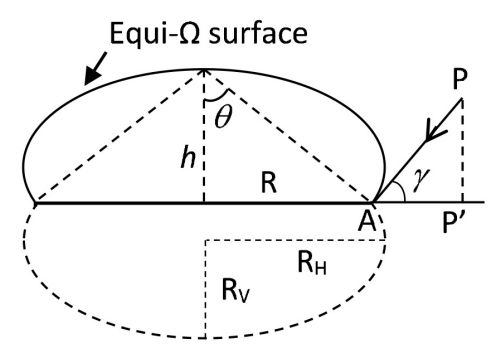

(a)

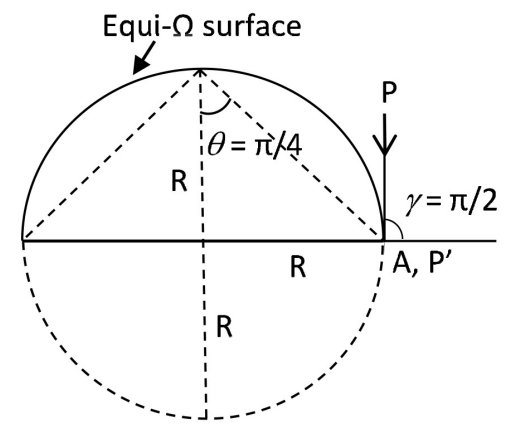

(b)

Figure 19. The simple case of a right cone: (a): The case of a general $\gamma$. (b): The case of $\gamma=\pi / 2$.

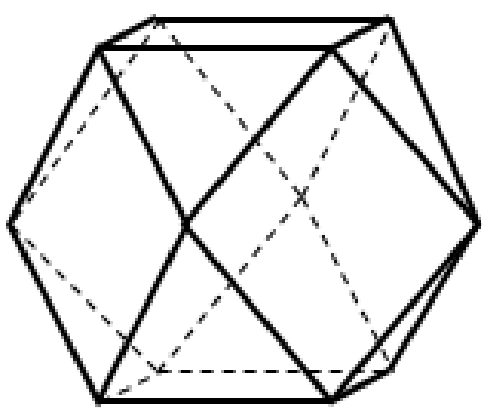

Figure 20. Flux of the electric field of a point charge at the center of a non-regular polyhedron through its polygonal faces.

$$
\Omega_{t}=\left(4 \pi-6 \Omega_{s}\right) / 8=0.5513
$$

By using $[\mathrm{XV}]$, we have finally:

$$
\Phi_{s}=0.10817 Q / \varepsilon_{0}
$$

and

$$
\Phi_{\tau}=0.04387 Q / \varepsilon_{0}
$$

It would be possible to calculate $\Omega_{t}$ directly by the geometry as we did in the case of the square face. We easily find, for this case, $\cos \theta=\sqrt{2} / \sqrt{3}$.

Using Equation (3):

$$
\Omega_{t}=2 \pi\{1-(3 / \pi) \arctan [(\sqrt{2} / \sqrt{3}) \tan (\pi / 3)]\}=0.5513
$$

That is the same previous result.

\subsection{Linear, Surface and Volume Ratios between Inscribed and Circumscribes Polyhedrons}

By simple inspection, we see that the linear ratio between a regular polyhedron inscribed and another similar one circumscribed to the same sphere is $R_{L}=\cos \theta, \theta$ being the angle of a straight regular pyramid with its vertex being the center of the polyhedron and whose base is the face of it. The ratios be- 
tween the areas and the volumes of the two polyhedrons are respectively $R_{A}=\cos ^{2} \theta$ and $R_{V}=\cos ^{3} \theta$.

From Equation (9) we can take $\cos \theta$.

$$
\cos \theta=\tan \left[\left(2 \pi-\Omega_{F}\right) /(2 N)\right] / \tan (\pi / N)
$$

Using Equation (23), where $\Omega_{F}$ is the solid angle for one face, it is possible to calculate the area ratio $R_{A}$ and volume ratio $R_{V}$ between the inscribed and circumscribed polyhedrons for all five regular polyhedrons:

Tetrahedron: $N=3 ; \Omega_{F}=\pi \quad \therefore \quad R_{L}=\cos \theta=1 / 3, R_{A}=1 / 9$ and $R_{V}=1 / 27$

Cube: $N=4 ; \quad \Omega_{F}=2 \cdot \pi / 3 \quad \therefore \quad R_{L}=\cos \theta=1 / \sqrt{3}, \quad R_{A}=1 / 3$ and $R_{V}=\sqrt{3} / 9$

Octahedron: $N=3 ; \quad \Omega_{F}=\pi / 2 \quad \therefore \quad R_{L}=\cos \theta=1 / \sqrt{3}, \quad R_{A}=1 / 3$ and $R_{V}=\sqrt{3} / 9$

Dodecahedron: $N=5 ; \quad \Omega_{F}=\pi / 3 \quad \therefore \quad R_{L}=\cos \theta=\sqrt{3} /[3 \tan (\pi / 5)]$, $R_{A}=1 /\left[3 \tan ^{2}(\pi / 5)\right]$ and $R_{V}=\sqrt{3} /\left[9 \tan ^{3}(\pi / 5)\right]$

Icosahedron: $N=3 ; \Omega_{F}=\pi / 5 \quad \therefore \quad R_{L}=\cos \theta=\tan (3 \pi / 10) / \sqrt{3}$, $R_{A}=\tan ^{2}(3 \pi / 10) / 3$ and $R_{V}=\sqrt{3} \tan ^{3}(3 \pi / 10) / 9$.

We remember that:

$$
\begin{gathered}
\tan (\pi / 5)=\sqrt{5-2 \sqrt{5}} \\
\tan (3 \pi / 10)=\sqrt{(5+2 \sqrt{5}) / 5}
\end{gathered}
$$

It is interesting to note that the cube and octahedron have both the same value of those ratios.

These results for the five regular polyhedrons are very hard to get if we do not use pyramid solid angles.

\section{Conclusion}

The starting point of the solid angle for an isosceles triangle, the Fundamental Formula of the present work, is clearly very useful to study many solid angle related problems. In special, Equation (2) and Equation (20) are important results in the study of equi- $\Omega$ surfaces. The Fundamental Formula is also a very convenient way for the determination of a solid angle of a closed curve when the observer is on the curve itself. Its results, as the analysis of equi- $\Omega$ surfaces, are not presented in the literature.

\section{Conflicts of Interest}

The author declares no conflicts of interest regarding the publication of this paper.

\section{References}

[1] de Pinho, A.G. and Amaral, L.C.M. (1968) Note on the Determination of Branching-Ratios by the Sum-Coincidence Method. Anais da Academia Brasileira de 
Ciências, 40, 111, 1968.

[2] Amaral, L.C.M. (1968) Emprego do Método de Coincidência-soma no Estudo de Esquemas de Desintegração. Master Degree Thesis, Pontifícia Universidade Católica do Rio de Janeiro, Rio de Janeiro, Brasil.

[3] Koveshnikov, P. (2017) Right N-Sided Pyramid Solid Angle. https://math.stackexchange.com/questions/2465971/right-n-sided-pyramid-solid-a ngle

[4] Cell, J.W. (1941) Solid Angles. The American Mathematical Monthly, 42, 136-138. https://doi.org/10.2307/2303605

[5] Van Oosterom, A. and Strackee, J. (1983) The Solid Angle of a Plane Triangle. IEEE Transactions on Biomedical Engineering, BME-30, 125-126.

https://doi.org/10.1109/TBME.1983.325207 